\title{
Estimation of Right Ventricular Volume without Geometrical Assumptions Utilizing Cardiac Magnetic Resonance Data
}

\author{
R Battani ${ }^{1}, \mathrm{C} \mathrm{Corsi}^{1}{ }^{1}$, A Sarti ${ }^{1}, \mathrm{C}$ Lamberti ${ }^{1}, \mathrm{~T} \mathrm{Piva}^{2}, \mathrm{R} \mathrm{Fattori}^{2}$ \\ ${ }^{1}$ D.E.I.S. - University of Bologna, Italy \\ ${ }^{2}$ Department of Radiology, S.Orsola-Malpighi University Hospital, Bologna, Italy
}

\begin{abstract}
Object of this study is to provide a new method to estimate right ventricular volume. The right ventricle $(R V)$ is a complex crescent-shaped structure and, contrary to the left ventricle, it cannot be suitable with any simple geometric model existing in medical literature. To test $R V$ volume estimation based on level set method, we performed $R V$ endocardial surface detection on reconstructed cardiac Magnetic Resonance (MR) studies. Level set algorithm has been integrated into a package for $3 D$ reconstruction of medical images, navigation and processing. The semi-automatic procedure has been applied to end-systolic (ES) and enddiastolic (ED) frames of MRI data belonging to 5 patients affected by dilative and infiltrative pathologies and 5 affected by congenital heart diseases. This study demonstrates the feasibility of accurate evaluation of $R V$ chamber size and shape, even in case of irregular geometry.
\end{abstract}

\section{Introduction}

Right ventricular volume (RVV) is a cardiac function parameter whose importance is increasingly recognized especially in pathological cases involving infants and young children. Magnetic Resonance Imaging is becoming a valuable tool in clinical practice, especially in cardiological diagnosis, because it provides clear images of the cardiac structures and shows anatomic details that echocardiographic investigations cannot evidence.

Artifacts and distortions due to differences in magnetic susceptibility of adjacent tissues and little inhomogeneities in the static magnetic field give difficulties in quantitative analysis of MR cardiac images. The identification of endocardial borders is also influenced by the presence of evident papillary muscles and trabeculations.

Contrary to the left ventricle, for which the exploiting of simple geometric models, such as rotational ellipsoid, is possible, a geometric description of the $\mathrm{RV}$ is more difficult. Attempts were tried in the past, approximating the RV cavity with a semi-ellipsoid or modeling it as the difference between two ellipsoids (ellipsoidal shell model) [1]. However an accurate model for RV endocardial surface requires mathematical assumptions, whose complexity makes the procedure of extraction of the shape and assessment of correlated parameters unsuitable in terms of correctness and processing time. In the common clinical practice, medical doctors use variations of the multi-planar Simpson's rule, to extract ventricular volumes, independently from cavity shape. Manual or semi-automatic contour detection (edge extraction, active contours, snakes) of the endocardial walls on 2D short axis slices obtained from the MR study is performed. The area of each segmented region is calculated in pixels, whose dimensions are known, and multiplied by slice thickness. Ventricular volume quantification is generally obtained adding these multiple slice volumes and correction factors can be used to keep volume assessments more precise.

In this study we decided to apply level sets to RV segmentation to test their capability to adapt to complex shapes. In fact the key features of level sets representation as compared to other deformable models are the ability to handle strong variations in topology and numerical stability.

\section{Method}

Our method is based on the $3 \mathrm{D}$ reconstruction of a MR cardiac study, throughout the cardiac cycle, stored in DICOM files. A semi-automatic procedure to detect endocardium in $3 \mathrm{D}$ space is applied on the reconstructed volume using level set algorithm.

\subsection{The tool}

To perform the estimation of RVV through a simple and fast procedure, we use our software based on 3D data visualization. This tool has been developed using the Visualization Toolkit (VTK), an open-source objectoriented $\mathrm{C}++$ library for computer graphics [2]. Its peculiarity is the complete portability and the integration with the scripting language Tcl/Tk. The employment of 
different languages allows exploiting their best potentialities: data structures and algorithms can be developed in $\mathrm{C}++$, where specification of highly complex computational details is simple and immediate, while a window-oriented interface can be realized in $\mathrm{Tcl} / \mathrm{Tk}$ quickly and efficiently (Figure 1).

The result is a clear graphical user interface (GUI) with accessible commands and windows for navigation of $3 \mathrm{D}$ data through the simple use of keyboard and mouse controls.

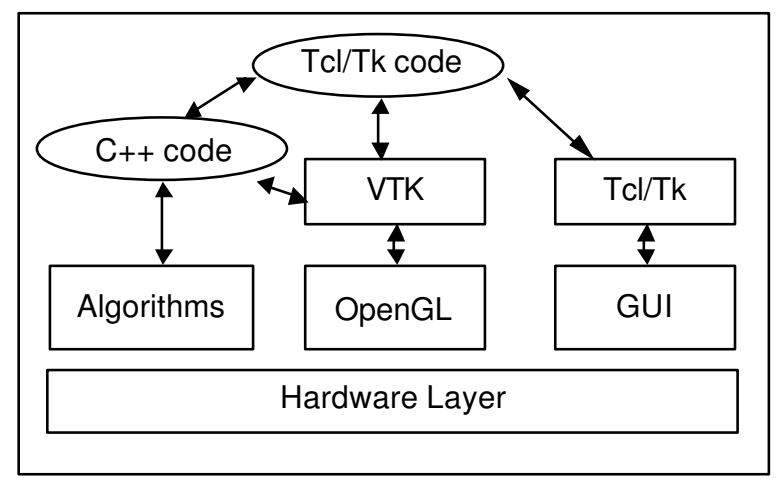

Figure 1. Logical layers of the software.

\subsection{Level Set Method}

The geometric deformable models with regularizers or level sets, introduced by Osher and Sethian [3,4], are numerical techniques used to track the evolution of interfaces that have been introduced by Malladi in the medical vision community for segmentation of anatomical structures [5].

The capability to adapt to complex shapes is the main reason that led us to test its application to $\mathrm{RV}$ segmentation. The main idea of this method is to embed the deformable model in a higher dimensional space. The surface that evolves in time is represented during the evolution as the zero level set of a function

$$
\Psi(\mathbf{x}, t): \mathfrak{R}^{3} \longrightarrow \mathfrak{R}(t)=\left\{\mathbf{x} \in \mathfrak{R}^{3} \mid \Psi(\mathbf{x}, t)=0\right\}
$$

that is necessarily a closed surface.

Given an initial surface $\gamma_{0}$, the hypersurface is defined as $\Psi(\overline{\mathbf{x}})=\operatorname{dist}\left(\mathbf{x}, \gamma_{0}\right)$ where dist is the signed Euclidean distance between a point and the surface. The evolution of the surface $\gamma$ is guided by a partial differential equation involving the level set function:

$$
\frac{\partial \Psi}{\partial t}+F|\nabla \Psi|=0
$$

The speed function $F$ used to control shape recovery process in the image $\mathrm{I}(\mathbf{x})$ is: with

$$
F=g \varepsilon \kappa-\beta \nabla g \cdot \frac{\nabla \Psi}{|\nabla \Psi|}
$$

$$
g=\left[1+\left(\frac{\left|\nabla\left(G_{\sigma} \otimes I(\mathbf{x})\right)\right|}{\alpha}\right)^{2}\right]^{-1}
$$

In the expression of $F$ the first term is an interface tension force that depends on the curvature ? and the second term is a force that attracts the interface towards the boundaries. The edge indicator $g$ is a non-increasing function of the gradient of a smoothed version of the initial image. The parameter $\alpha$ selects the contrast of the objects we have to consider in the image during the motion of the embedding, and the variance $\sigma$ of the gaussian $G$ defines the size of the smallest features we desire to detect in the image. The parameter $\beta$ is used to limit the regularization of the embedding controlled by the parameter $\varepsilon$.

The resulting equation of the motion for the level set function $\phi(\mathbf{x}, \mathrm{t})$ is:

$$
\frac{\partial \Psi}{\partial t}+g \varepsilon \kappa|\nabla \Psi|-\beta \nabla g \cdot \nabla \Psi=0
$$

The narrow band level set method [6] has been implemented to make the algorithm more efficient.

Once the evolution will have a steady state solution when the geometry dependent terms balances the advection term, we obtain the RV endocardial surface.

The volume estimate is obtained applying the divergence theorem on the final detected surface.

\subsection{The segmentation procedure}

The procedure for the RV segmentation consists of one step only: the user selects the most characteristic slices of the 3D dataset, obtained by cutting the dataset with planes perpendicular to the ventricular long axis, with $1 \mathrm{~mm}$ step. The operator places some points near the expected contour of the endocardial wall.

The software joins the points placed on the image to create a polygon and for each polygon it calculates the signed distance function. The zero levels of the signed distance function are interpolated between the slices to obtain a 3D initial condition (Figure 2, left).

This initialization can be saved and modified afterwards if not satisfying. The level set algorithm leads the evolution of the initialization.

The final result is the surface corresponding to right ventricular cavity. Figure 2 shows the result (on the right) of the evolution of a 3D initial condition (on the left). 

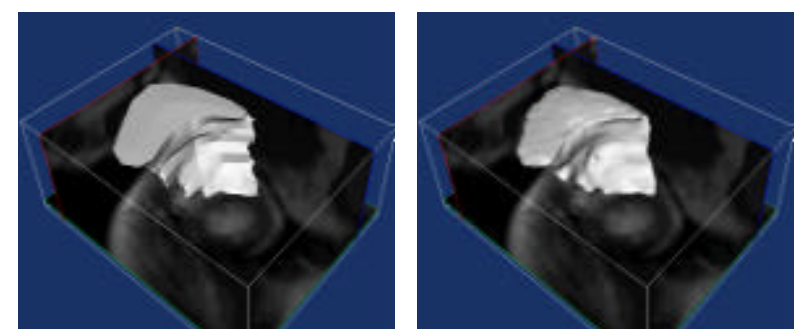

Figure 2. Initial condition (on the left) and extracted surface (on the right) superimposed on original 3D data.

Superimposing the extracted surface on the original data, also in dynamic mode, allows the user to evaluate the correctness of the $3 \mathrm{D}$ detection and to evidence the presence of a possible pathology (Figure 3).

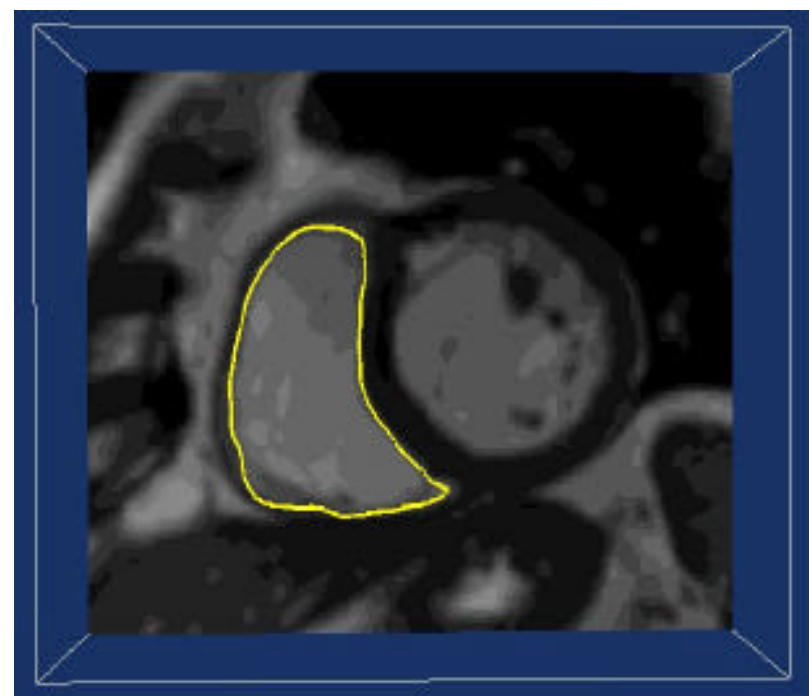

Figure 3. Contouring superimposed on original 3D data.

\subsection{Data analysis}

Five patients affected by dilative and infiltrative pathologies (hypertrophic cardiomyopathy and amyloidosis), and five patients affected by congenital heart disease (transposition of the great arteries, tetralogy of Fallot) were imaged using a GE Signa Infinity 1.5 Tesla MRI system. A fast-CINE acquisition of 11 short axis slices was performed at $20 \mathrm{~Hz}$ frame rate with ECG and respiratory gating $(8 \mathrm{~mm}$ slice thickness, $8 \mathrm{~mm}$ spacing between slices).

The tool allows the user to choose a DICOM directory containing an entire MR study. The directory usually includes a cardiac cycle. Our software automatically reconstructs a $3 \mathrm{D}$ dataset from $2 \mathrm{D}$ slices and provides its visualization and animation throughout the cycles.

2D MR slices are 256x256 pixels images with 16-bits grayscale values. The reconstructed $3 \mathrm{D}$ data presents a voxel spacing of $1.7 \times 1.7 \times 1 \mathrm{~mm}$.
Different display modes allowed the observer to slice the $3 \mathrm{D}$ dataset using planes without losing the perception of the three dimensions. Structural details were evidenced also by properly regulating brightness and contrast of the images. Surface and volume rendering provided a qualitative view of the whole dataset or only of a region of interest

A typical standard methodology was employed to select the right ventricular cavity properly and to avoid wrong estimates: if the pulmonary valve was visible, only the portion of the right ventricular outflow tract below the level of the pulmonary valve was included in the volume. The papillary muscles were considered inside the extracted surface. Difficulties were encountered in the evaluation of the apical region in the MR image because of the uncertain interface between blood and endocardium.

3D data were browsed and rendered by medical doctors. RV volume estimations were performed for the entire cardiac cycle applying level set model, following the previously described methodology.

Medical doctors manually outlined RV endocardial contours on each of the selected slices of the MR study and applied multi-planar Simpson's rule to obtain reference volume estimates. We compared these estimates with those performed by three observers applying our software procedure for ED and ES frames.

A linear regression analysis was performed on data. In order to obtain a more meaningful data interpretation, additional parameters describing the accuracy and the variability of the method were also extracted applying the Bland-Altman analysis [7].

\section{Results}

Medical doctors judged the endocardium surfaces detected using our software reliable and effective. 3D navigation and animation of the chamber clearly evidenced the presence of the investigated pathologies (Figure 4).

The number of the required slices to obtain a reliable segmentation is small (about $10 \%$ of the short axis slices). The application of a 30 iterations algorithm requires less than 15 seconds on a standard PC (Pentium II processor $755 \mathrm{MHz}, 512 \mathrm{Mb}$ RAM).

A highly significant relationship was found between $\mathrm{RV}$ volumes (range 50-350 ml) measured by medical doctors $(\mathrm{x})$ and the volumes obtained applying our algorithm based on level set method $(y)(y=0.99 x+0.36$, $\mathrm{r}=0.99$, Standard Error of the Estimate $\mathrm{SEE}=8.29 \mathrm{ml}$, mean error \pm Standard Deviation $\Delta \pm \mathrm{SD}=-0.14 \pm 8.09 \mathrm{ml}$, $\mathrm{n}=20$ ) (Figure 5). The level of significance was $\mathrm{p}<0.0001$. For a given RV volume, determination of $95 \%$ confidence limits would predict the true volume range of $\pm 8.09 \mathrm{ml}$ 

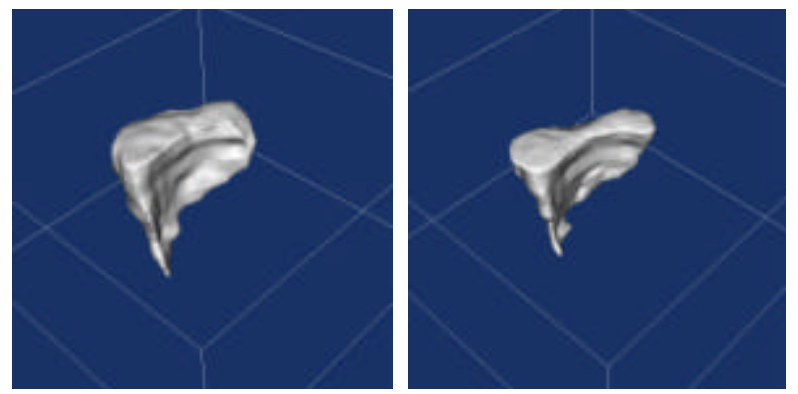

Figure 4. Segmented ED and ES frames (46-years-old female patient affected by hypertrophic cardiomyopathy).

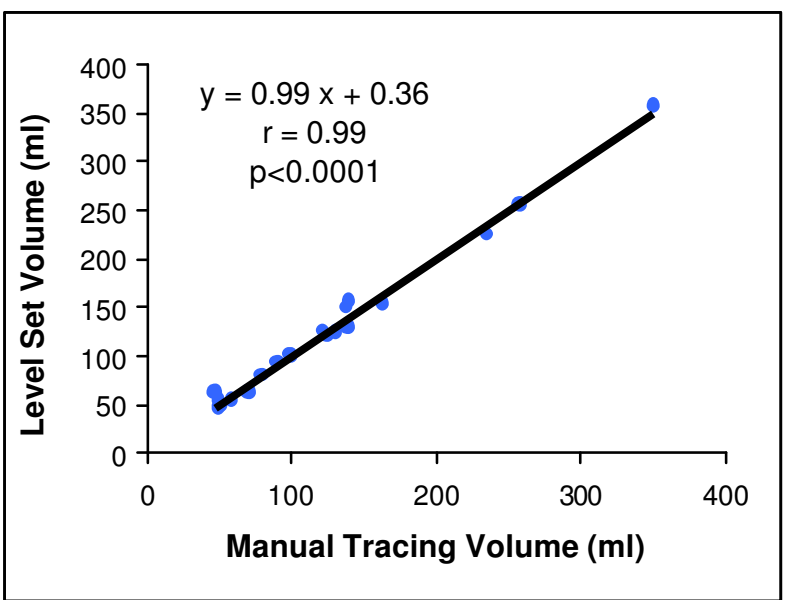

Figure 5. Correlation curve between manual tracing and our method.

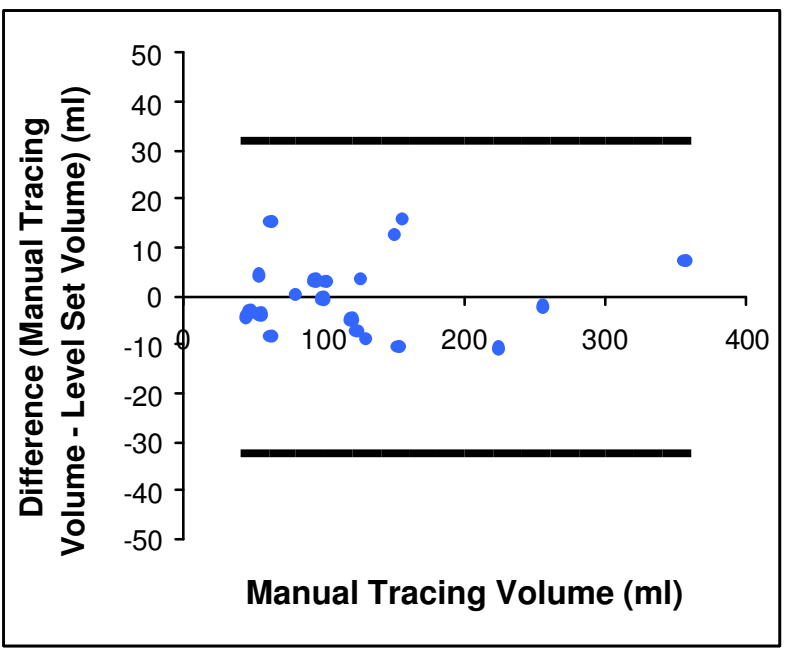

Figure 6. Bland-Altman analysis.

\section{Discussion and conclusions}

MR images allow a reliable and fast qualitative and quantitative diagnosis of the congenital heart disease, such as tetralogy of Fallot and transposition of the great arteries. MR investigation is also important after surgical treatment because patients may develop right ventricular dysfunction and serial assessment of RV function is required in long-term clinical follow-up. For these reasons, medical doctors show a strong necessity of practical and light tools to investigate the right ventricle in a fast and accurate way, in particular to extract RV volume and its related parameters, such as the stroke volume and ejection fraction.

A method for RV volume assessment, based on 3D semi-automatic surface detection with level sets, has been developed. The method allows quick and accurate evaluation of chamber size and shape, even in case of irregular geometry due to pathological conditions.

Considerably more work remains to do in validating this approach with data acquired by other imaging techniques.

\section{Acknowledgements}

UE FSE has provided a grant for the development of this work under Spinner program.

We would like to thank the Department of Radiology, S.Orsola-Malpighi University Hospital, Bologna, for providing the data.

\section{References}

[1] Frangi A, Niessen WJ, Viergever MA. Three-dimensional modeling for functional analysis of cardiac images: a review. IEEE transactions on medical imaging 2001;20:225.

[2] Visualization Toolkit website, http://www.vtk.org.

[3] Osher S, Sethian JA. Fronts Propagating with curvature dependent speed: Algorithm based on Hamilton Jacobi formulation. J Comp Phys 1988;79:12-49.

[4] Sethian JA. Level Set Methods and Fast Marching Methods, Cambridge: University Press, 1999.

[5] Malladi R, Sethian JA, Vemuri BC. Shape Modeling with Front Propagation: A Level Set Approach. IEEE Trans on Pattern Analysis and Machine Intelligence 1995;17:158 175.

[6] Adalsteinsson D, Sethian JA. A Fast Level set Method for Propagating Interfaces. J Comp Phys 1994;112:334-363.

[7] Bland M, Altman DG. Statistical methods for assessing agreement between two methods of clinical measurement. Lancet 1986;i:307-310.

Address for correspondence.

Claudio Lamberti.

DEIS, Università degli Studi di Bologna

Viale Risorgimento 2, 40136 Bologna, Italy.

E-mail: clamberti@deis.unibo.it. 